\title{
Molecular Interplay between microRNA-34a and Sirtuin1 in Hyperglycemia-Mediated Impaired Angiogenesis in Endothelial Cells: Effects of Metformin ${ }^{\mathrm{s}}$
}

\author{
Gnanapragasam Arunachalam, Arun Prasath Lakshmanan, Samson Mathews Samuel, \\ Chris R. Triggle, and Hong Ding \\ Departments of Pharmacology (G.A., A.P.L., S.M.S.) and Medical Education (C.R.T., H.D.), Weill Cornell Medicine-Qatar, \\ Education City, Doha, Qatar (C.T.)
}

Received June 22, 2015; accepted November 10, 2015

\begin{abstract}
Impaired angiogenesis is a prominent risk factor that contributes to the development of diabetes-associated cardiovascular disease. MicroRNAs (miRNAs), small noncoding RNAs, are implicated as important regulators of vascular function, including endothelial cell differentiation, proliferation, and angiogenesis. In silico analysis and in vitro studies indicate that silent information regulator 1 (SIRT1) is a potential target for endothelial cell-specific miRNAs. In this study, we investigated the molecular crosstalk between miR-34a, the protein product of SIRT1 (sirtuin1), and the antidiabetic drug, metformin, in hyperglycemia-mediated impaired angiogenesis in mouse microvascular endothelial cells (MMECs). MMECs were cultured, transfected with either a miR-34a inhibitor or mimic in normal glucose $(11 \mathrm{mM})$ or high glucose $(\mathrm{HG}, 40 \mathrm{mM})$ in the presence or absence of metformin. The expression of miR-34a,
\end{abstract}

sirtuin1, and their signaling targets was evaluated. miR-34a expression is upregulated in a hyperglycemic milieu and parallels changes in the expression of sirtuin1, post-translational modification of endothelial nitric oxide synthase (phospho/acetylation), as well as an impairment in angiogenesis. The presence of metformin, or the inhibition of miR-34a using an anti-miR-34a inhibitor, increases the expression of sirtuin 1 and attenuates the impairment in angiogenesis in HG-exposed MMECs. In contrast, overexpression of a miR-34a mimic prevents metformin-mediated protection. These data indicate that miR-34a, via the regulation of sirtuin1 expression, has an anti-angiogenic action in MMECs, which can be modulated by metformin. In summary, miR-34a represents both a target whereby metformin mediates its vasculoprotective actions and also a potential therapeutic target for the prevention/treatment of diabetic vascular disease.

\section{Introduction}

The global increase in the prevalence of type 2 diabetes (T2DM) and the link to an elevated risk for both cardiovascular disease (CVD) and cancer are major concerns that heighten the need for improved approaches for the early detection, prevention, and treatment of T2DM (Tremblay and Hamet, 2015). Vascular endothelial cells (ECs) play a major role in maintaining normal cardiovascular homeostasis, and endothelial dysfunction is recognized as an early indicator of diabetes-associated CVD (Ding and Triggle, 2010; Eelen et al., 2015). The diabetic milieu, namely hyperglycemia, excess free fatty acid release, and insulin resistance, initiates a sequence of events in the vasculature that includes oxidative stress, impaired endothelial function, increased

The work was supported by National Priorities Research Program grants [4910-3-244, 5-149-3-040, 6-428-3-113] from Qatar National Research Fund (a member of Qatar Foundation) and a Biomedical Research Program establishment grant. The statements made herein are solely the responsibility of the author(s).

dx.doi.org/10.1124/jpet.115.226894.

S This article has supplemental material available at jpet.aspetjournals.org. inflammation, and impaired angiogenesis (Avogaro et al., 2011; Roberts and Porter, 2013; Sena et al., 2013). Despite the known high risk for patients with diabetes to develop CVD, the pathogenesis underlying the relationship between T2DM and CVD is not fully understood. Therefore, the identification of new molecular targets that are associated with the development of vascular dysfunction in diabetes is of high scientific interest.

MicroRNAs (miRNAs) are short ( 22 nucleotides) noncoding RNAs that typically bind to 3 ' untranslated regions of mRNAs, acting principally at the post-transcriptional level and repressing the mRNA translation and stability (Olson, 2014). miRNAs have been implicated as key elements playing crucial roles in the pathogenesis of diabetes-associated CVD, including endothelial dysfunction, angiogenesis, hypertrophy, and heart failure (Urbich et al., 2008; Leeper and Cooke, 2011; Hata, 2013; Beltrami et al., 2014; Arunachalam et al., 2015; Zhu and Leung, 2015). Several endothelial-specific miRNAs have been identified as regulators of cardiac and endothelial function and linked to the regulation of angiogenesis and the development of senescence and inflammation (Suárez and Sessa, 2009; Caporali et al., 2011; Staszel et al., 2011;

ABBREVIATIONS: AMPK, AMP-activated protein kinase; Ang-1, angiopoetin-1; Ang-2, angiopoetin-2; CVD, cardiovascular disease; DMEM, Dulbecco's modified Eagle's medium; EC, endothelial cell; eNOS, endothelial nitric oxide synthase; HG, high glucose; miRNA, microRNA; MMEC, mouse microvascular EC; NG, normal glucose; SIRT1, silent information regulator 1; T2DM, type 2 diabetes; TSP-1, thrombospondin-1. 
Zampetaki and Mayr, 2012; Arunachalam et al., 2015). MicroRNA-34a (miR-34a) is highly expressed in ECs and has been reported to play a key role in the regulation of EC proliferation, inflammation, senescence, and apoptosis (Qin et al., 2012; Badi et al., 2014; Fan et al., 2015). Elevated miR-34a expression has been reported in senescent human umbilical vein ECs as well as in the heart and spleen of aged mice (Ito et al., 2010).

Silent information regulator 1 (SIRT1), referred to as an anti-ageing gene, has been described as a novel regulatory switch in vascular EC homoeostasis (Potente et al., 2007; Potente and Dimmeler, 2008). Furthermore, sirtuin1, the protein product of SIRT1, via the deacetylation of endothelial nitric oxide synthase (eNOS), increases the generation of nitric oxide and enhances endothelium-dependent vasodilatation (Mattagajasingh et al., 2007; Arunachalam et al., 2010). miR-34a directly binds to SIRT1 through a miR-34a binding site within the 3 ' untranslated regions of SIRT1, inhibits the expression of the protein sirtuin1, and regulates apoptosis via the sirtuin1-p53 pathway (Yamakuchi et al., 2008). In human umbilical vein ECs, the overexpression of miR-34a downregulates sirtuin 1 expression and induces EC senescence, whereas the knockdown of miR-34a enhances the expression of sirtuin 1 and attenuates endothelial senescence (Ito et al., 2010). In addition, several studies have highlighted miR-34a as an important mediator of ageing-related cardiac dysfunction by modulating the expression of sirtuin 1 (Tabuchi et al., 2012; Boon et al., 2013; Han et al., 2015). Furthermore, the overexpression of miR-34a in endothelial progenitor cells reduces the expression of sirtuin 1 expression, resulting in senescence and impaired angiogenesis (Zhao et al., 2010).

Metformin is the first-line oral antidiabetic drug reported to protect endothelial function and the cardiovascular system against the impact of diabetes (UK Prospective Diabetes Study Group, 1998; Sena et al., 2011; Arunachalam et al., 2014; Triggle and Ding, 2014). The assumed primary target for mediating the antidiabetic actions of metformin is AMPactivated protein kinase (AMPK); however, other targets have been implicated, and a potential link between the therapeutic benefits of metformin and the modulation of miRNAs has also been reported and may explain the apparent benefits of metformin in reducing the incidence of certain types of cancer (Blandino et al., 2012; Foretz et al., 2014). However, although the relative importance of sirtuin1, miR-34a, and metformin in the regulation of endothelial function is recognized, the interplay between them and their roles in modulating the effects of hyperglycemia on endothelial function has not been exploited. In the present study, we have investigated the interactions between miR-34a and sirtuin1, as well as miR$34 \mathrm{a}$, as a potential therapeutic target for metformin in the attenuation of hyperglycemia-mediated impaired angiogenesis in mouse microvascular ECs (MMECs).

\section{Materials and Methods}

Chemicals. Unless otherwise stated, all chemicals used were of analytical grade and purchased from Sigma-Aldrich (St. Louis, MO).

EC Culture. MMECs, originated from mouse pancreas, were purchased from American Type Culture Collection (Manassas, VA) and cultured in Dulbecco's modified Eagle's medium (DMEM) consisting of either normal glucose (NG; $11 \mathrm{mM})$ or high glucose (HG; $40 \mathrm{mM}$ ), supplemented with $5 \%$ fetal bovine serum at $37^{\circ} \mathrm{C}$ in a humidified atmosphere containing $5 \% \mathrm{CO}_{2}$, as previously described (Arunachalam et al., 2014). Glucose concentrations were chosen on the basis of random glucose measurements taken from normoglycaemic mice that approximates $11 \mathrm{mM}$, and for type 2 diabetic, $\mathrm{db} / \mathrm{db}$ mice, which, for the latter, have been reported to range from 30 to $55 \mathrm{mM}$ and as also detailed in The Jackson Laboratory (Bar Harbor, ME) Phenome Data Summary for mice (Pannirselvam et al., 2002; Semeniuk et al., 2002). For the studies comparing the effects of mannitol, MMECs were incubated with media that consisted of $11 \mathrm{mM}$ D-glucose plus $29 \mathrm{mM}$ D-mannitol.

miR-34a Inhibition and Overexpression. MMECs were grown in antibiotic-free DMEM for 24 hours. For the protocols involving miR34a inhibition, MMECs at 30-50\% confluence were transfected with a miR-34a inhibitor (small, synthetic, and chemically modified singlestranded RNA molecules designed to specifically bind to and inhibit the mature miRNAs from functioning) or a miRNA inhibitor negative control (25 nmol/l, mirVana; Life Technologies Waltham, MA) for 12 hours using Opti-MEM reduced serum media and lipofectamine-2000 according to the manufacturer's instructions (Invitrogen, Waltham, MA). After 12 hours of transfection, the transfection media was changed into media consisting of either NG or HG for another 48 hours (media was changed at 24 hours). To overexpress miR-34a in MMECs, cells were transfected with the miR-34a mimic (small, synthetic, and chemically modified double-stranded RNAs that mimic particular endogenous miRNAs and enable miRNA functional analysis by upregulation of miRNA activity) or the negative control mimic ( $50 \mathrm{nmol} /$, mirVana; Life Technologies) for 12 hours using Opti-MEM reduced serum media and lipofectamine-2000, according to the manufacturer's instructions (Invitrogen). After 12 hours of transfection, the media was changed into a media consisting of either NG or HG for another 48 hours (media was changed at 24 hours). At the end of the experimental period, lysates were taken for further analysis.

Treatment with Metformin. MMECs were cultured in culture media (DMEM) that consisted of either NG or HG alone or with metformin (50 $\mu \mathrm{M}, 1,1$-dimethylbiguanide hydrochloride; SigmaAldrich) for 48 hours. For the miR-34a overexpression studies, media was changed after 12 hours of transfection to either NG or HG with or without metformin.

Quantitative Real-Time Polymerase Chain Reaction Analysis. Enriched miRNAs were isolated from experimental samples using miRNA isolation kits, according to the manufacturer's instructions (Qiagen, Valencia, CA). Quantitative real-time polymerase chain reaction was performed with the mirVana quantitative real-time polymerase chain reaction miRNA amplification kit (Life Technologies). Amplification and detection of specific products were performed using TaqMan primers (miR-34a and U6; Life Technologies), according to the manufacturer's recommended protocol with the ABI StepOne Fast Real-Time PCR System (Applied Biosystems, Foster City, $\mathrm{CA}$ ), and the reaction was performed in triplicates. The U6 small nucleolar RNA was used as the housekeeping small RNA reference gene, and the relative gene expression was normalized to the expression of U6 small nuclear RNA.

Immunoblotting. Immunoblotting was used to detect the expression of sirtuin1, Phosphorylated eNOS (peNOS) ( $\operatorname{Ser}^{1177}, \mathrm{Thr}^{495}$ ), eNOS, Ac-Lys, angiopoietein-1 (Ang-1), angiopoietein-2 (Ang-2), and thrombospondin-1 (TSP-1), as previously described (Arunachalam et al., 2014). Briefly, cellular protein $(50 \mu \mathrm{g})$ was electrophoresed on SDS-PAGE gel and transblotted onto nitrocellulose membrane. Membranes were blocked with $5 \%$ (w/v) nonfat milk or bovine serum albumin in phosphate-buffered saline containing $0.1 \%(\mathrm{v} / \mathrm{v})$ Tween 20 and incubated with the appropriate primary antibody (1:1000 dilution). After washing, bound antibody was detected using antirabbit/mouse antibody (1:2000 dilution) linked to horseradish peroxidase, and bound complexes were detected and documented using enhanced chemiluminescence method (Bio-Rad, Hercules, CA) and Geliance Imaging System (PerkinElmer, Waltham, MA).

eNOS Acetylation by Immunoprecipitation. Whole-cell lysate was used for eNOS immunoprecipitation, as previously described 
(Arunachalam et al., 2010). An antibody against eNOS (1:40 dilution; Cell Signaling, Danvers, MA) was added to $100 \mu \mathrm{g}$ cellular proteins in a final volume of $400 \mu \mathrm{l}$, and incubated for 1 hour. Protein-A/G agarose beads ( $20 \mu \mathrm{l}$; Santa Cruz Biotechnology, Santa Cruz, CA) were added to each sample and kept overnight at $4^{\circ} \mathrm{C}$ on a rocker. The beads were washed three times and then resuspended in $40 \mu \mathrm{l}$ radioimmunoprecipitation assay buffer. The immunoprecipitated eNOS agarose bead suspension was resolved by 7.5\% SDS-PAGE. To assess the acetylation of eNOS, the membranes were first probed against acetyl lysine antibody (mouse monoclonal acetyl lysine; Cell Signaling) and then reprobed for eNOS (rabbit anti-eNOS; Cell Signaling).

In Vitro Angiogenesis (Tube Formation) Assay. The in vitro angiogenic activity of MMECs was determined by Matrigel tube formation assay. Briefly, after the experimental period described above, MMECs were stained with cell-permeable dye, calcein ( $2 \mathrm{mg} / \mathrm{ml}$; Invitrogen), for 30 minutes and replated in 24-well plates precoated with $150 \mu \mathrm{l} /$ well growth factor-reduced Matrigel (Geltrex; Invitrogen) and incubated at $37^{\circ} \mathrm{C}$ in cell culture incubator. After 12 hours of incubation, capillary-like tube formation was observed with a computer-assisted microscope (EVOS, Thermo Fisher Scientific, MA). Tube formation was defined as a tube-like structure exhibiting a length four times its width. Images of tube morphology were taken in 10 random microscopic fields per sample at original magnification, $10 \times$. The numbers of branch points in duplicate wells were counted and averaged using ImageJ software (National Institutes of Health).

Statistical Analysis. All data were analyzed by statistical software GraphPad Prism 5.0 (San Diego, CA). Statistical analysis was performed by using one-way analysis of variance. Post hoc comparisons between the groups were performed by Tukey's multiple comparisons test. Results are presented as mean \pm S.E.M. with $P<0.05$ used to indicate statistical significance.

\section{Results}

Hyperglycemia-Mediated miR-34a Induction Causes Changes in Expression of Sirtuin1, eNOS, and Impaired Angiogenesis in ECs. Exposure of MMECs to HG for 48 hours showed a significant increase in miR-34a expression, and there was no significant difference in miR-34a expression compared with the osmotic control (11 $\mathrm{mM}$ glucose plus $29 \mathrm{mM}$ mannitol) (Fig. 1A). Therefore, we have investigated whether endothelial dysfunction in HG-exposed MMECs can be prevented by decreasing the expression of miR-34a to that comparable in NG-cultured MMECs using a miR-34a inhibitor. MMECs were transfected with either a miR-34a inhibitor or a scrambled control and incubated with HG and NG for 48 hours. As shown in Fig. 1B, the miR-34a inhibitor significantly decreased the expression of miR-34a in both NG- and HG-treated MMECs when compared with scrambled control $(P<0.05)$. To further investigate the effects of miR-34a inhibition on ECs, we examined the expression levels of sirtuin1, eNOS, and eNOS phosphorylation. As shown in Fig. 1, C-G, comparing with NG, the exposure of
A
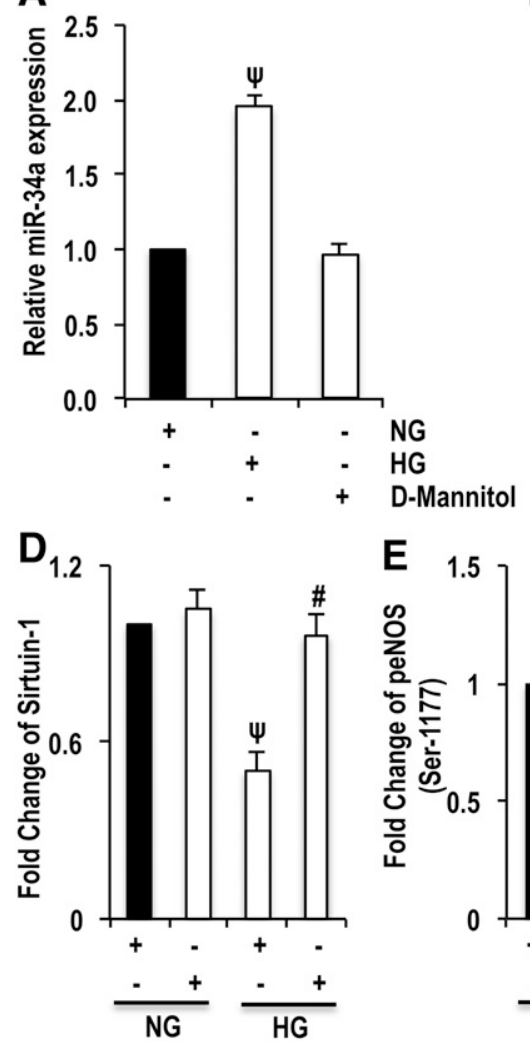

B

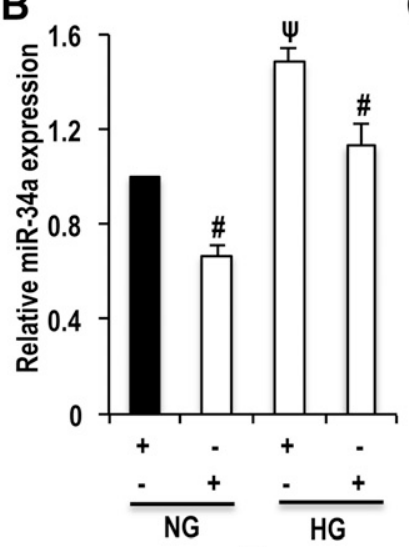

C

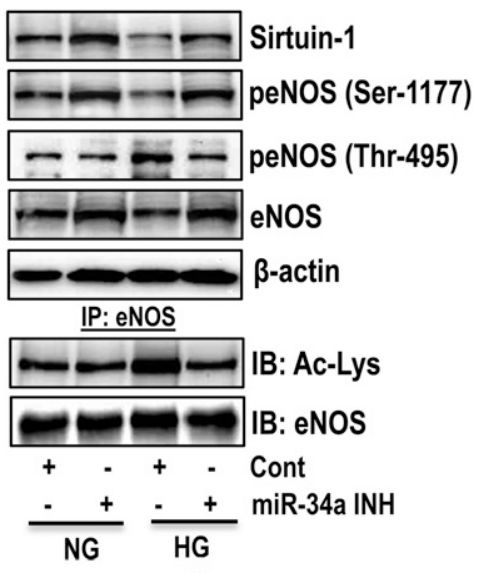

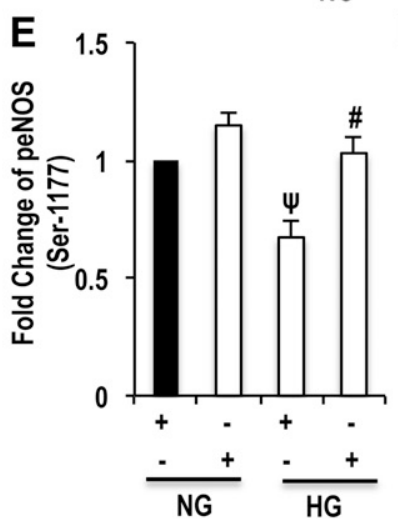

$\mathbf{F}$
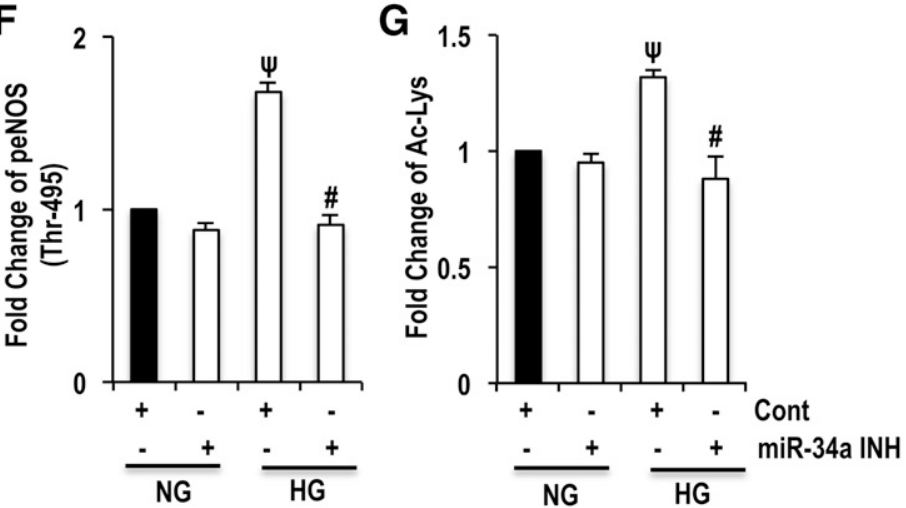

Fig. 1. Effect of exposure to HG on expression of miR-34a, sirtuin1, and eNOS in ECs. MMECs (B-G) with the exception of (A) (no transfection) were transfected with either scrambled control or a miR-34a inhibitor, and, after 12 hours of transfection, cells were exposed to media consisting of either NG $(11 \mathrm{mM})$ or HG $(40 \mathrm{mM})$ for another 48 hours. Cell lysates were subjected to analyses of miR-34a expression by quantitative real-time polymerase chain reaction and sirtuin1, peNOS (Ser ${ }^{1177}, \mathrm{Thr}^{495}$ ), eNOS, Ac-Lys, and $\beta$-actin by immunoblotting. (A and B) Histogram values represent mean \pm S.E.M. of relative miR-34a expression (normalized to U6 small nuclear RNA, $n=4$ ). $(\mathrm{C}-\mathrm{G})$ Representative gel images and histogram values represent mean \pm S.E.M. of relative intensity of sirtuin1, peNOS ( $\left.\operatorname{Ser}^{1177}, \mathrm{Thr}^{495}\right)$, and acetylated eNOS levels $(n=4) .{ }^{*}(P<0.05)$, significant when compared with NG. ${ }^{\#}(P<0.05)$, significant when compared with cells transfected with control scrambled miRNA. 
MMECs to HG (that were transfected with scrambled control) resulted in a significant $(P<0.05)$ reduction of sirtuin1 expression, which was paralleled by a decrease in eNOS phosphorylation ( $\mathrm{Ser}^{1177}$ ), increased eNOS phosphorylation $\left(\mathrm{Thr}^{495}\right)$, and increased eNOS acetylation. Inhibition of miR34-a in HG-treated MMECs transfected with the miR34 a inhibitor resulted in a significant $(P<0.05)$ increase in sirtuin1 expression together with enhanced peNOS level $\left(\right.$ Ser $\left.^{1177}\right)$, reduced eNOS acetylation, and eNOS phosphorylation at $\mathrm{Thr}^{495}$.

Based on the finding that sirtuin1 and eNOS downregulation were associated with impaired angiogenesis in ECs, we further examined the effects of miR-34a inhibition on the expression of the effectors/mediators of angiogenesis Ang-1, Ang-2, and TSP-1. As shown in Fig. 2, A and C-E, the exposure to $\mathrm{HG}$ resulted in a significant $(P<0.05)$ reduction in Ang-1 expression along with significant $(P<0.05)$ increases in Ang-2 and TSP-1 levels in MMECs compared with MMECs exposed to NG after both transfected with scrambled control miRNA. Furthermore, MMECs transfected with the miR-34a inhibitor and maintained in HG showed significant increases in Ang-1 expression along with a decrease in Ang-2 and TSP-1 levels compared with those transfected with scrambled control. Importantly, tube-forming activity (Fig. 2, B and F) was also significantly impaired in MMECs that were transfected with negative control miRNA in HG when compared with MMECs maintained in NG; however, MMECs transfected with the miR-34a inhibitor in HG showed a significant $(P<0.05)$ increase in tube-forming activity versus those transfected with the scrambled control. Thus, these results indicate that,
A

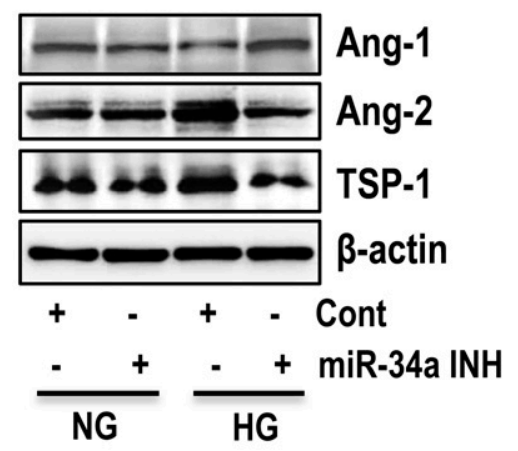

B
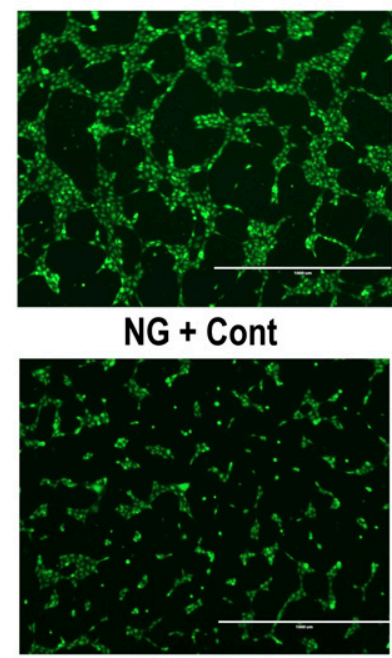

HG + Cont
C

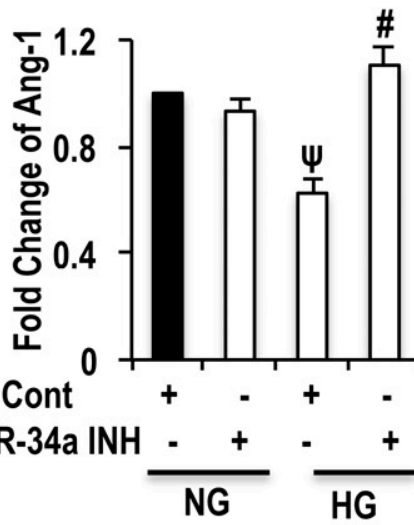

D

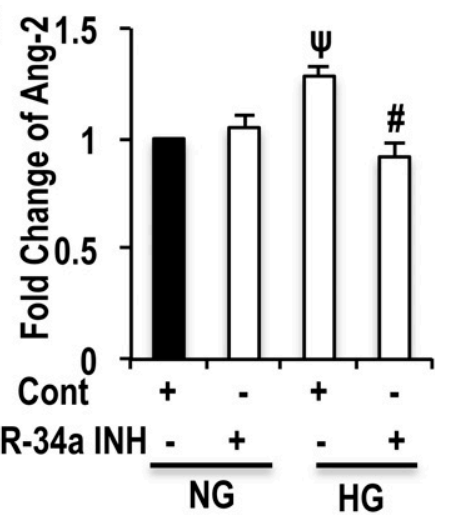

$\mathrm{E}$

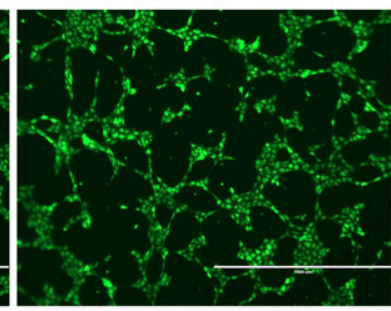

NG + miR-34a INH

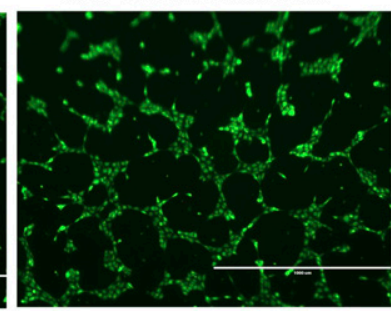

$H G+$ miR-34a INH

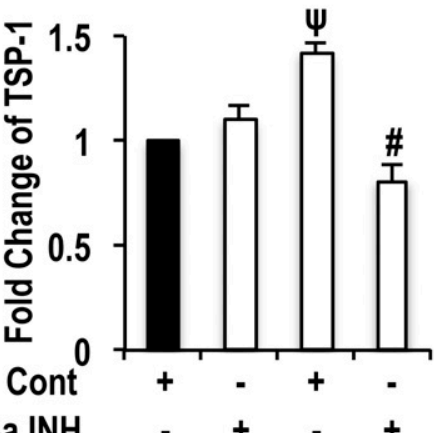

miR-34a INH $\frac{-+}{\mathrm{NG}} \frac{-}{\mathrm{HG}}$

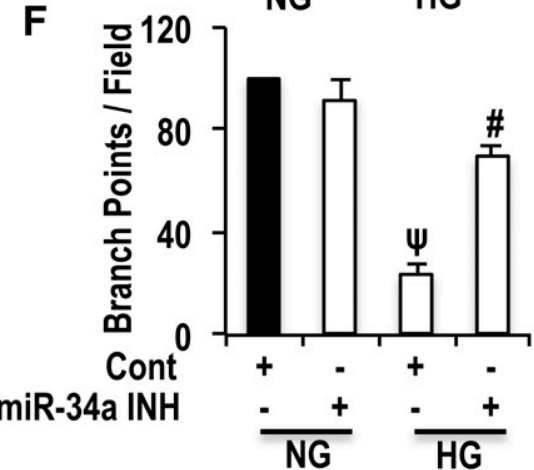

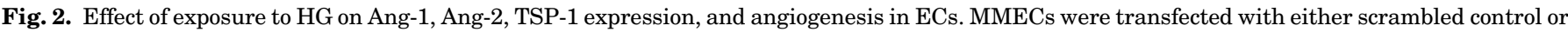

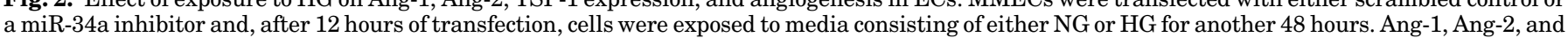

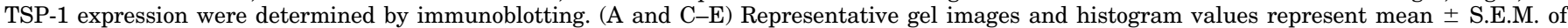

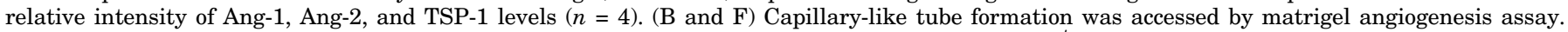

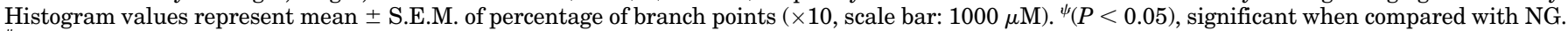
\# $(P<0.05)$, significant when compared with cells transfected with control scrambled miRNA. 
in MMECs, miR-34a induction by HG results in a reduction in the expression of sirtuin 1 and phosphorylation of eNOS along with impaired angiogenesis that can be reversed by inhibition of miR-34a.

Metformin Modulates miR-34a, Sirtuin 1, and eNOS, and Attenuates Hyperglycemia-Mediated Impaired Angiogenesis in ECs. We have previously reported that metformin attenuates hyperglycemia-induced endothelial senescence via a mechanism that involves the upregulation of sirtuin 1 and its downstream signaling pathway in MMECs (Arunachalam et al., 2014). However, it is not known whether miR-34a mediates the protective effects of metformin on sirtuin 1 expression and EC function in HG. To investigate this potential relationship, MMECs were cultured in media containing either NG or $\mathrm{HG}$ alone or with metformin for 48 hours, and changes in the expression of miR-34a, sirtuin1, and eNOS were assessed by quantitative immunoblotting. As shown in Fig. 3, miR-34a expression was significantly $(P<$ 0.05) increased in MMECs exposed to HG for 48 hours with a parallel reduction in sirtuin1 and eNOS phosphorylation levels as well as a subsequent increase in eNOS acetylation, as previously shown (Fig. 1). When MMECs were exposed to HG together with metformin, there was a significant $(P<$ 0.05 ) reduction in the expression of miR-34a accompanied by a parallel increase in the expression of sirtuin1 and eNOS phosphorylation at Ser ${ }^{1177}$, as well as a decrease in the acetylation of eNOS when compared with MMECs exposed to HG alone $(P<0.05)$.

To further investigate the interplay between metformin, miR-34a and endothelial function experiments were designed to determine the effects of the metformin-mediated reduction in miR-34a on the effectors/mediators of angiogenesis: namely Ang-1, Ang-2, and TSP-1. As shown in Fig. 4, A and C-E, exposure of MMECs to HG resulted in a significant $(P<0.05)$ reduction in Ang-1 expression along with an increase in Ang-2 and TSP-1 expression when compared with MMECs exposed to NG. In contrast, MMECs exposed to HG together with metformin showed a significant $(P<0.05)$ increase in Ang-1 expression with diminished Ang-2 and TSP-1 expression. Tube-forming activity (Fig. 4, B and F) was also decreased in MMECs exposed to HG when compared with MMECs exposed to NG, whereas MMECs exposed to HG together with metformin showed a significant increase in tube-forming activity. These results indicate that metformin reduces miR$34 \mathrm{a}$ expression, increases the expression of sirtuin1, and enhances eNOS phosphorylation at $\mathrm{Ser}^{1177}$, as well as attenuating hyperglycemia-induced impaired angiogenesis.

Overexpression of miR-34a Reduces the MetforminMediated Attenuation of Impaired Angiogenesis in HG-Exposed ECs. To further investigate whether the endothelial-protective action of metformin was miR-34adependent, the effect of metformin in MMECs wherein miR$34 \mathrm{a}$ had been overexpressed using a miR-34a mimic was examined in NG versus HG conditions. In MMECs transfected with a miR-34a mimic and exposed to either NG or HG, there was a significant increase in miR-34a expression, which remained elevated despite the presence of metformin, whereas, in comparison, in MMECs transfected with scrambled control, there was a reversal of HG-induced upregulation of miR-34a expression after treatment with metformin $(P<$
A

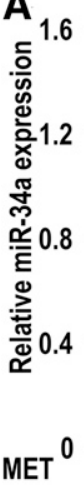

+

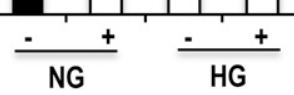

B

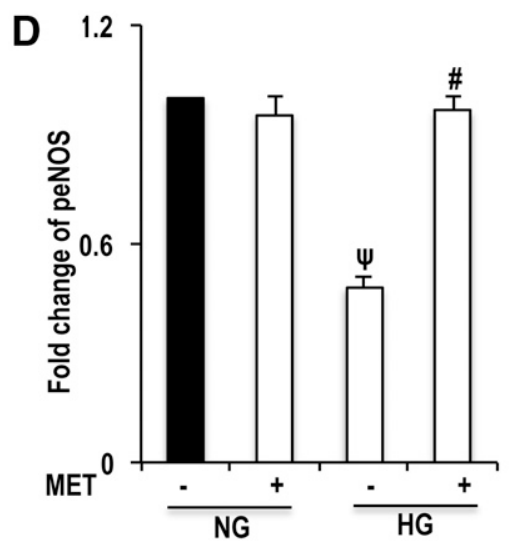

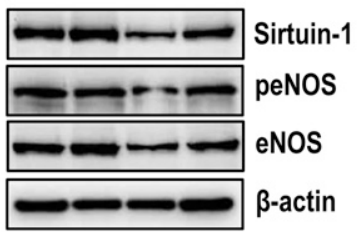

IP: eNOS

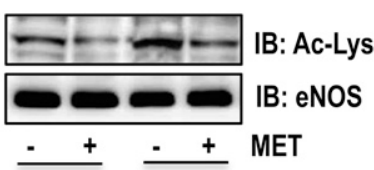

MET

C

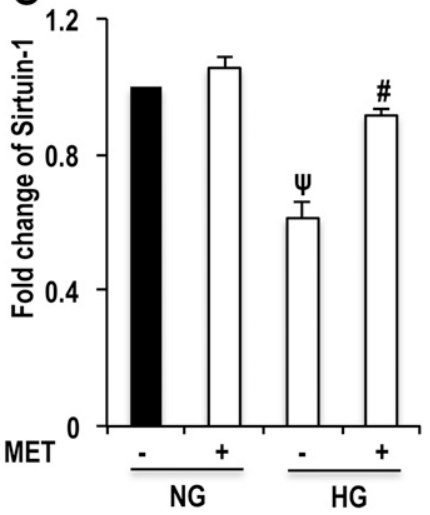

E 1.6

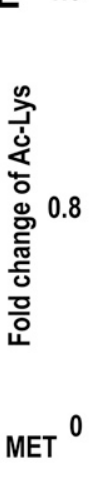

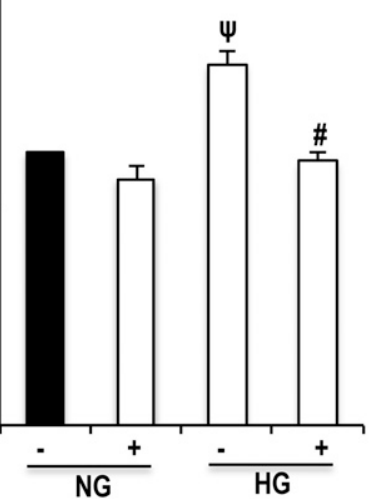

Fig. 3. Effect of treatment with metformin on expression of miR-34a, sirtuin1, and eNOS in ECs exposed to HG. MMECs were cultured in media consisting of either NG or HG for 48 hours in the presence or absence of metformin $(50 \mu \mathrm{M})$. Cell lysates were subjected for analyses of miR-34 expression by quantitative realtime polymerase chain reaction and sirtuin1, peNOS (Ser ${ }^{1177}$ ), eNOS, Ac-Lys, and $\beta$-actin by immunoblotting. (A) Histogram values represent mean \pm S.E.M. of relative miR-34a expression (normalized to U6 small nuclear RNA, $n=4)$. (B-E) Representative gel images and histogram values represent mean \pm S.E.M. of relative intensity of sirtuin1, peNOS, and acetylated eNOS levels $(n=4) . \psi * *(P<$ $0.05)$, significant when compared with NG. \# $(P<0.05)$, significant when compared with HG alone. 


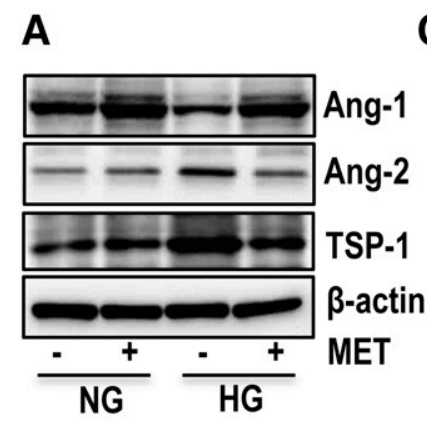

B

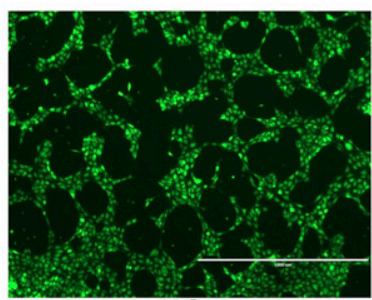

NG

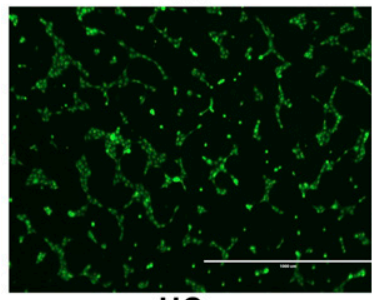

HG

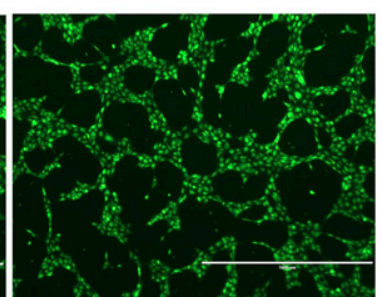

NG + MET

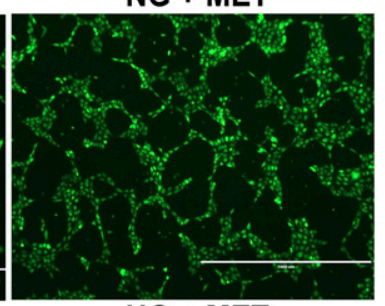

HG + MET
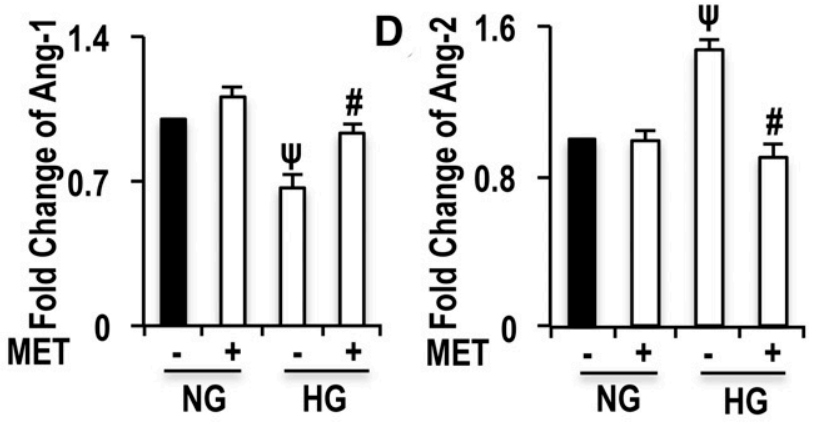

E
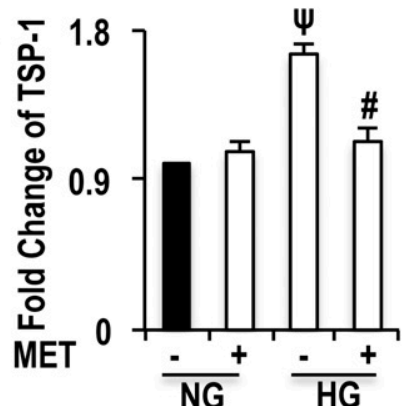

F

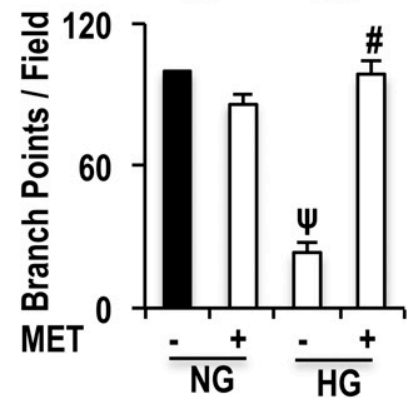

Fig. 4. Effect of treatment with metformin on expression of Ang-1, Ang-2, TSP-1, and angiogenesis in ECs. MMECs were cultured in media consisting of either NG or HG for 48 hours in the presence or absence of metformin $(50 \mu \mathrm{M})$. The expression of Ang-1, Ang-2, and TSP-1 was determined by immunoblotting. (A and C-E) Representative gel images and histogram values represent mean \pm S.E.M. of relative intensity of Ang-1, Ang-2, and TSP-1 levels $(n=4)$. (B and F) Capillarylike tube formation was accessed by matrigel angiogenesis assay. Histogram values represent mean \pm S.E.M. of percentage of branch points $(\times 10$, scale bar: $1000 \mu \mathrm{M})$. $\psi(P<0.05)$, significant when compared with NG. ${ }^{\#}(P<0.05)$, significant when compared with HG alone.
0.05) (Fig. 5A). Similarly, reductions in the expression of sirtuin1 and levels of eNOS phosphorylation at Ser ${ }^{1177}$ following the exposure of MMECs to HG (transfected with scrambled control) were also attenuated by the addition of metformin, but, again, the transfection of a miR-34a mimic in MMECs resulted in the negation of the protective effects of metformin on expression of sirtuin 1 and eNOS phosphorylation (Fig. 5, B-D).

We further investigated the effects of metformin in MMECs with an overexpression of miR-34a in the context of the expression of markers of angiogenesis and EC tube formation. As shown in Fig. 6, A and C-E, the exposure to HG resulted in a significant $(P<0.05)$ decrease in Ang-1 expression with concomitant increase in Ang-2 and TSP-1 expression comparing with NG in MMECs transfected with scrambled control, and these effects were reversed by metformin. Tube-forming activity was also significantly impaired in MMECs (transfected with scrambled control) exposed to HG, whereas treatment with metformin significantly $(P<0.05)$ increased tube formation, as reflected by the data depicted in Fig. 6, B and F. Furthermore, exposure of MMECs in which miR-34a was overexpressed to either NG or HG resulted in a decrease in Ang-1 and elevation of Ang-2 and TSP-1 levels. Moreover, in this protocol, tube formation remained impaired even in the presence of metformin. These results indicate that the overexpression of miR-34a effectively counteracts the protective effects of metformin against HG-induced impaired angiogenesis in ECs.

\section{Discussion}

The present study documents that treatment of MMECs with metformin inhibits the expression of miR-34a, with the subsequent increase in the levels of sirtuin1 and active phosphorylated eNOS at Ser $^{1177}$ leading to the restoration/ revival of the angiogenic capacity in HG-exposed MMECs. Diabetes/Hyperglycemia with subsequent oxidative stress has been implicated as the main cause of endothelial dysfunction and resultant cardiovascular disease (Ding and Triggle, 2010; Giacco and Brownlee, 2010). A healthy endothelium is important for the maintenance of angiogenesis; however, much less studied is the role of hyperglycemia in the development of aberrant angiogenesis, a key contributor to many diabetesrelated vascular pathologies, such as reduced wound-healing capacity, impaired coronary collateral vessel formation, higher rates of transplant rejections, and embryonic vasculopathy and ensuing cardiovascular complications (Kolluru et al., 2012).

In our study, we have investigated the angiogenic response of MMECs in NG and HG conditions; then delved into explaining the possible molecular mechanism through the involvement of miR-34a and its downstream effectors; and investigated whether we could reverse these effects with metformin - the most widely used antihyperglycemic drugwhich, based on data from both clinical and animal studies, has also been shown to protect endothelial function (Kinaan 


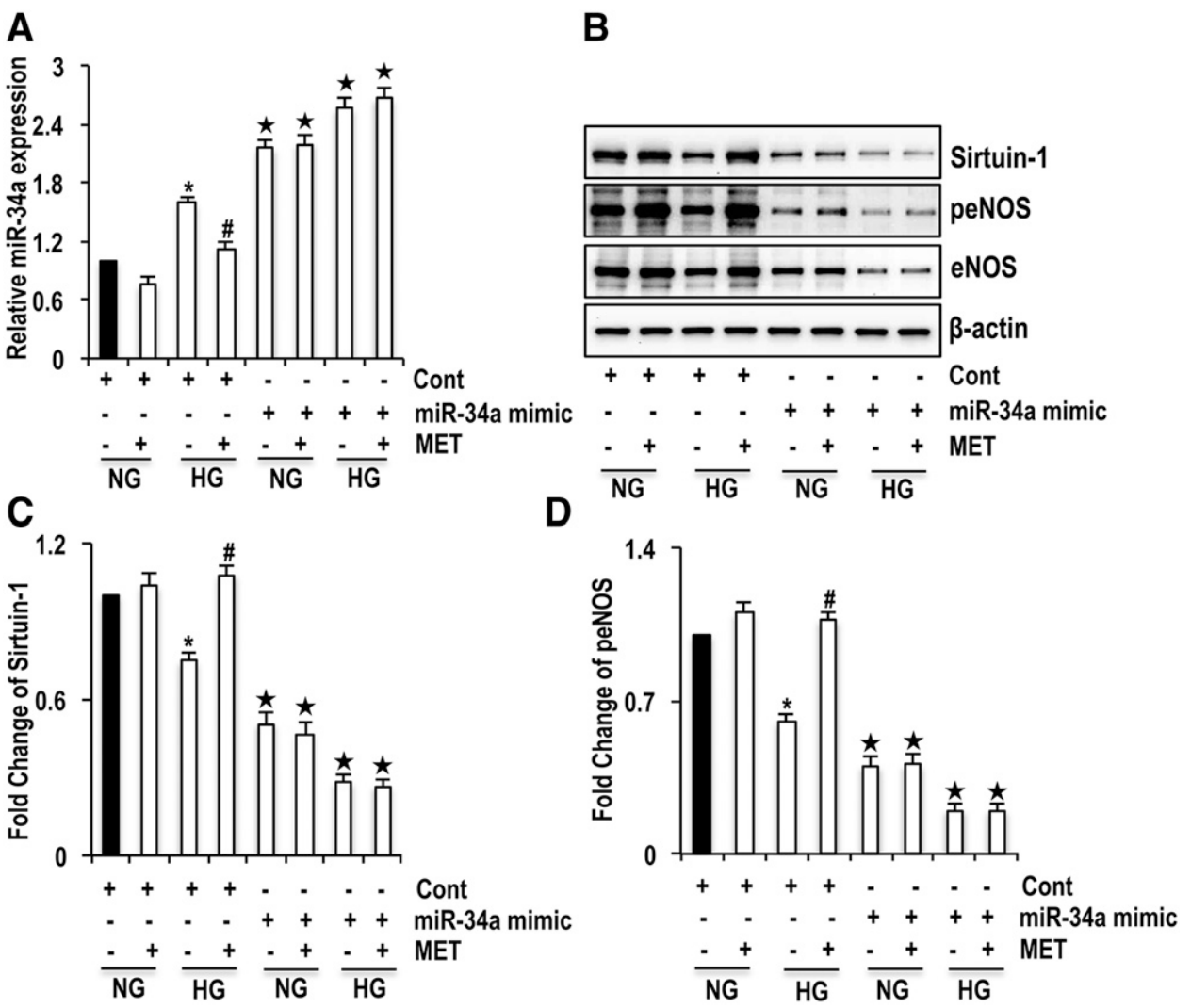

Fig. 5. Effects of overexpression of miR$34 \mathrm{a}$ on metformin-mediated regulation of the expression of sirtuin 1 and eNOS in ECs exposed to HG. MMECs were transfected with either scrambled control miRNA or a miR-34a mimic and, after 12 hours of transfection, cells were exposed to media consisting of either NG or HG for 48 hours in the presence or absence of metformin $(50 \mu \mathrm{M})$. Cell lysates were subjected for analyses of the expression of miR-34a by quantitative RT-PCR and sirtuin1, peNOS (Ser ${ }^{1177}$ ), and eNOS by immunoblotting. (A) Histogram values represent mean \pm S.E.M. of relative miR-34a expression (normalized to U6 small nuclear RNA, $n=4$ ). (B-D) Representative gel images and histogram values represent mean \pm S.E.M. of relative intensity of sirtuin 1 and peNOS levels $(n=4) . *(P<0.05)$, significant when compared with NG (transfected with control scrambled miRNA). ${ }^{\#}(P<0.05)$, significant when compared with $\mathrm{HG}$ alone (transfected with control scrambled miRNA $).{ }^{\star}(P<0.05)$, significant after transfection with a miR-34a mimic when compared with the counterparts transfected with control scrambled miRNA.

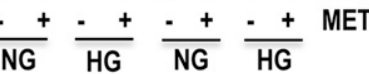

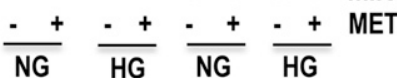

et al., 2015). Furthermore, miR-34a has been implicated as a tumor suppressor through the inhibition of angiogenesis (Yamakuchi et al., 2008). In addition, miR-34a has been shown to induce endothelial progenitor cell senescence and inhibit angiogenic capacity through the downregulation of sirtuin1 (Zhao et al., 2010). Data from cell-based studies and animal models indicate that sirtuin 1 is highly expressed in the vasculature and has been shown to function as a deacetylase for a number of vascular genes, including eNOS, and modulates diabetes, ageing, and CVD-associated vascular dysfunction (Orimo et al., 2009; D’Onofrio et al., 2015). More importantly, it has been reported that the loss of sirtuin 1 in ECs impairs migration and tube formation, thus limiting angiogenesis (Potente et al., 2007). Previously, we have shown that the decrease in sirtuin 1 levels correlated with the induction of EC senescence in HG-exposed MMECs (Arunachalam et al., 2014).

In our present study, we have seen a significant increase in the levels of miR-34a in HG-exposed MMECs (Fig. 1) with a significant decrease in the levels of sirtuin1 and peNOS $\left(\right.$ Ser $\left.^{1177}\right)$. Levels of peNOS ( $\mathrm{Thr}^{495}$ ) and acetylated eNOS, both of which negatively regulate eNOS activity (Nisoli et al., 2005), were found to be significantly higher in HG-exposed MMECs. Inhibition of the HG-induced increase in miR-34a expression (using a miR-34a inhibitor) reversed the effects of HG on the levels of sirtuin 1 and eNOS, indicating that the hyperglycemia-induced modulation of sirtuin1 levels and post-translational modification of eNOS operate through a miR-34a-dependent gene-regulatory mechanism.

The interactions between angiopoietins (Ang-1 and Ang-2) and their endothelium-specific tyrosine kinase receptor Tie2 are critical in the regulation of angiogenesis and vascular integrity (Fukuhara et al., 2010). Activation of Ang-1 and Tie2 signaling increases EC migration, sprouting, and angiogenesis, whereas activation of Ang-2 expression (an antagonist for Ang-1) inhibits Ang-1-mediated Tie2 stimulation and decreases EC migration, vascularization, and vessel maturation (Suri et al., 1998). Levels of proangiogenic Ang-1 decreased significantly, whereas levels of anti-angiogenic Ang-2 and TSP-1 increased significantly in HG-exposed MMECs (Fig. 2). The data from the current study indicate that the changes in these modulators of angiogenic potential result in a decrease in tubulogenic capacity, as evidenced by the marked decrease in the number of EC-derived branch points in our matrigel assay in the HG-exposed MMECs. However, inhibition of the HG-induced increase in miR-34a expression, by using a miR$34 \mathrm{a}$ inhibitor, significantly increased levels of Ang-1 while decreasing the levels of Ang-2 and TSP-1 (Fig. 2). Consequently, we have observed a significant increase in the number of EC-derived branch points in the HG-treated MMECs, which were also treated with the miR-34a inhibitor.

Metformin has been in clinical use for the treatment of T2DM for over 50 years, and its antidiabetic actions have been attributed to its inhibitory effect on mitochondrial complex 1 (El-Mir et al., 2000; Owen et al., 2000; Halimi, 2006; Madiraju et al., 2014). Preclinical in vivo studies and clinical data suggest that metformin possesses pleiotropic effects that protect the endothelium (Mather et al., 2001; Zhang et al., 2013; Arunachalam et al., 2014; Kinaan et al., 2015). However, the effect of metformin on miR-34a and its impact on endothelial function have not previously been studied. Treatment with metformin in HG-exposed MMECs reversed the HG-induced increase in miR-34a expression (Fig. 3) and subsequently increased the levels of sirtuin 1 and peNOS 
A
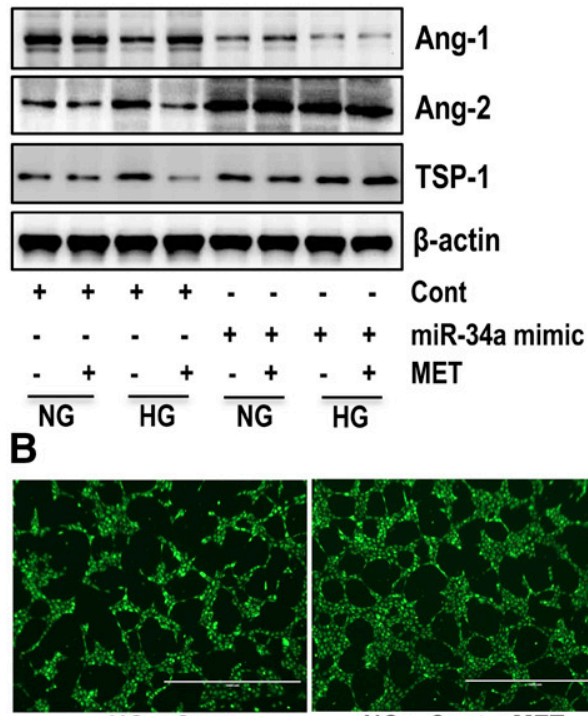

$\mathrm{NG}+$ Cont

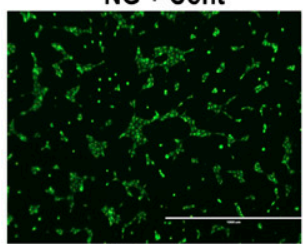

HG + Cont

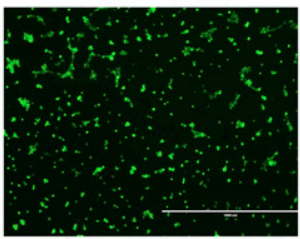

NG + miR-34 mimic

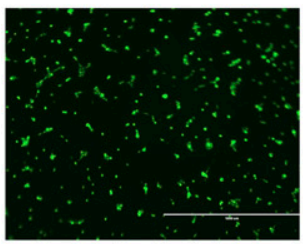

HG + miR-34 mimic
C

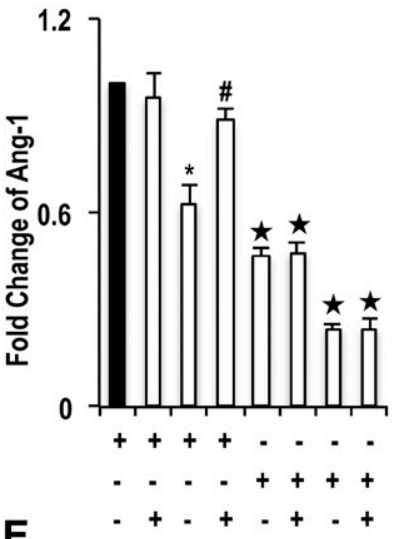

E

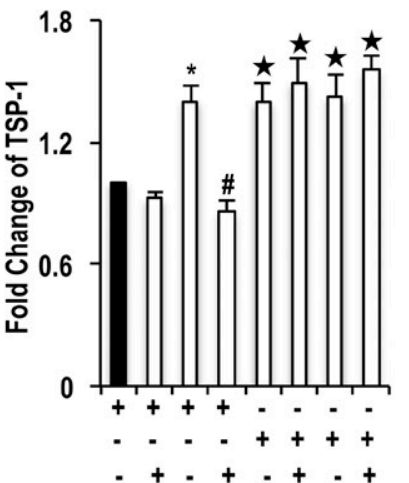

D

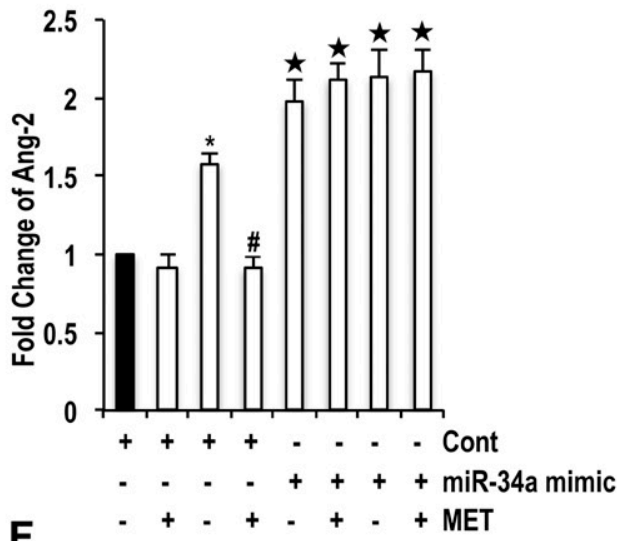

125

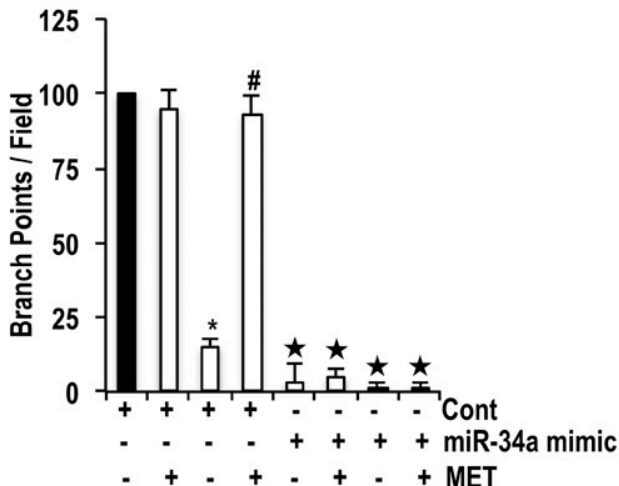

\section{G}

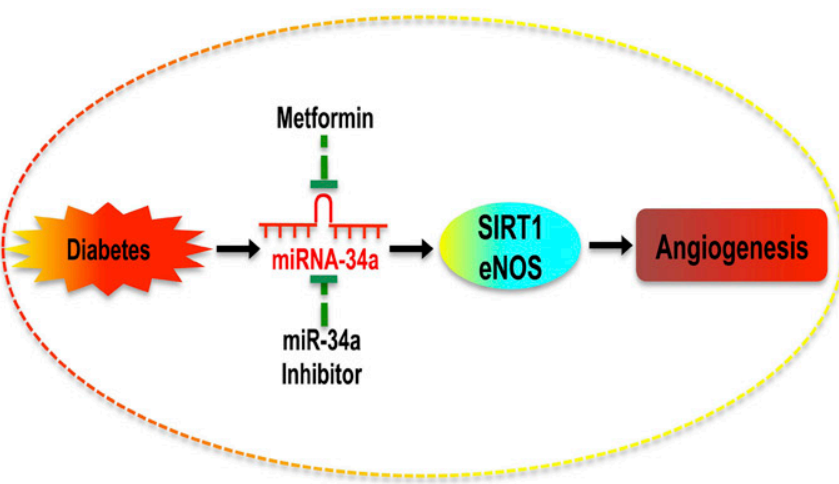

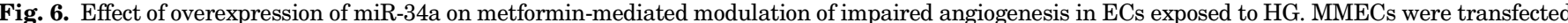

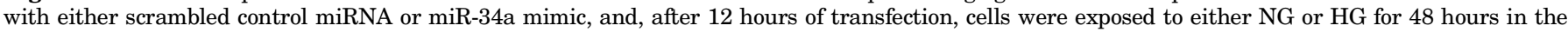

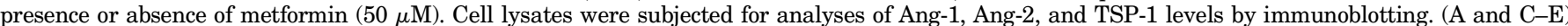

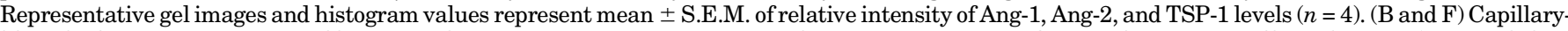

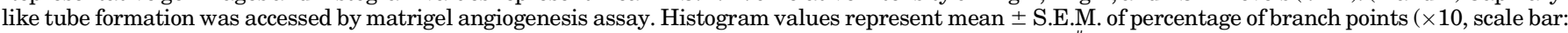

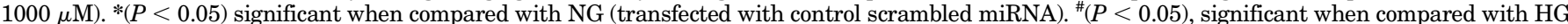

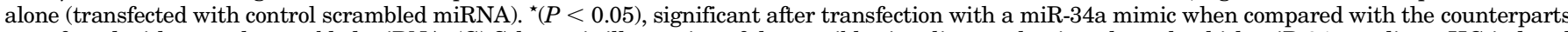

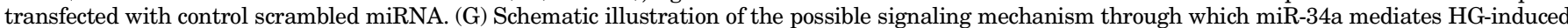

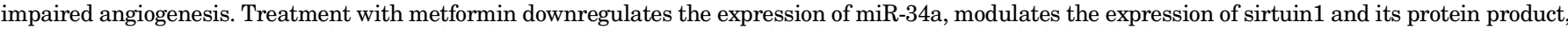
sirtuin1, and attenuates hyperglycemia-induced impaired angiogenesis and diabetes-associated endothelial dysfunction.

$\left(\right.$ Ser $\left.^{1177}\right)$ and decreased levels of acetylated eNOS, indicating that miR-34a is a molecular target for metformin through which it confers endothelial protection. Furthermore, the effects of metformin were mimicked by the miR-34a inhibitor. Similar comparative effects, a decrease in miR-34a expression with increases in sirtuin1 and peNOS levels, were also observed when MMECs were exposed to resveratrol in HG conditions (Supplemental Fig. 1).

A reduction and/or inhibition of sirtuin1 expression in ECs increase eNOS acetylation, which in turn reduces the generation of nitric oxide, thus leading to endothelial dysfunction. Conversely, sirtuin 1 promotes endotheliumdependent vasodilation by targeting eNOS for deacetylation and enhances the bioavailability of NO, increasing cell proliferation and angiogenesis (Nisoli et al., 2005; Mattagajasingh et al., 2007; Potente et al., 2007; Arunachalam et al., 2010). In the present study, exposure of ECs to HG decreased the tubulogenic potential of MMECs possibly linked to the associated decrease in Ang-1 and increase in Ang-2 and TSP-1; treatment with metformin in HG-exposed MMECs increased 
the level of pro-angiogenic Ang-1 and decreased the levels of anti-angiogenic Ang-2 and TSP-1, thereby improving the tubulogenic potential (Fig. 4). These data are in support of previous reports that decreased Ang-1 with increased Ang-2 expression was associated with vascular damage in the brains of mice with T2DM (Cui et al., 2011).

We also further investigated the role of miR-34a in the regulation of angiogenesis using a miR-34a mimic. The presence of a miR-34a mimic in NG-exposed MMECs significantly decreased the levels of sirtuin1 and peNOS (Ser ${ }^{1177}$ ) (Fig. 5), as well as decreasing the levels of Ang-1 and increasing the levels of Ang-2 and TSP-1, and decreased the tubulogenic capacity of these MMECs (Fig. 6). This effect was similar to that observed under $\mathrm{HG}$ conditions in the absence of a miR-34a mimic. In the presence of a miR-34a mimic, metformin did not effectively reverse the HG-induced effects on sirtuin1, peNOS (Ser ${ }^{1177}$ ), and tubulogenic potential in MMECs, indicating that the endothelial-protective and angiogenic potential of metformin operates through a miR-34a-dependent regulation of downstream sirtuin1, eNOS, Ang-1, Ang-2, and TSP1 expression. In addition, our study further evaluated the possible involvement of the metabolic sensor, AMPK, the cellular fuel gauge, in mediating the cellular effects of metformin. Our data demonstrate that AMPK silencing rendered metformin treatment ineffective in modulating the levels of miR-34a, sirtuin1, and its downstream signaling in HG-exposed MMECs, and thus provides support for the AMPK-dependent action of metformin (Supplemental Fig. 1). Metformin has also been reported to increase SIRT1 level/activity, or, in part, via the upstream kinase, serine-threonine liver kinase B1/AMPK pathway, and thereby protect the retinal endothelium (Zheng et al., 2012). It has also become increasingly apparent that the regulation of AMPK and SIRT1 is interdependent (Ruderman et al., 2010). For instance, data from a recent study indicate that inhibition of miR-34a results in a sirtuin1/AMPKdependent attenuation of steatosis in hepatocytes and liver tissue of high-fat diet-fed mice, and that AMPK is an important modulator of miR-34a and its effects on sirtuin1 and its target genes (Ding et al., 2015). Collectively, these data and that from the current study indicate that miR34a is an important target for the prevention of diabetes-related vascular disease, but, nonetheless, that further studies are also required to better understand how the miR-34a and AMPK/sirtuin1 signaling pathways are regulated and dysregulated in diabetes.

An elevation in miR-34a expression has also been linked to reduced sirtuin 1 expression in obesity and arteries from aged human and mice (Li et al., 2011; Choi et al., 2013; Fu et al., 2014) and accelerated apoptosis of cardiomyocytes in neonatal and adult mouse hearts (Yang et al., 2015). In conclusion, and as summarized in Fig. 6G, our results demonstrate that hyperglycemia-mediated induction of miR-34a results in a reduction in sirtuin1, reduced eNOS function that results in impaired angiogenesis. In addition, our results provide mechanistic evidence that therapeutic intervention with metformin reverses hyperglycemiaassociated impaired angiogenesis possibly through the modulation of miR-34a levels, which in turn regulates sirtuin1, AMPK, and eNOS activity, thus shedding more light on potential new therapeutic approaches to combat diabetesassociated CVD.

\section{Acknowledgments}

The authors thank Suparna Ghosh for valuable technical assistance.

\section{Authorship Contributions}

Participated in research design: Arunachalam, Ding.

Conducted experiments: Arunachalam, Lakshmanan (for revised version).

Performed data analysis: Arunachalam, Lakshmanan (for revised version).

Wrote or contributed to the writing of the manuscript: Arunachalam, Samuel, Triggle, Ding.

\section{References}

Arunachalam G, Samuel SM, Marei I, Ding H, and Triggle CR (2014) Metformin modulates hyperglycaemia-induced endothelial senescence and apoptosis through SIRT1. Br J Pharmacol 171:523-535.

Arunachalam G, Upadhyay R, Ding H, and Triggle CR (2015) MicroRNA signature and cardiovascular dysfunction. J Cardiovasc Pharmacol 65:419-429.

Arunachalam G, Yao H, Sundar IK, Caito S, and Rahman I (2010) SIRT1 regulates oxidant- and cigarette smoke-induced eNOS acetylation in endothelial cells: role of resveratrol. Biochem Biophys Res Commun 393:66-72.

Avogaro A, Albiero M, Menegazzo L, de Kreutzenberg S, and Fadini GP (2011) Endothelial dysfunction in diabetes: the role of reparatory mechanisms. Diabetes Care 34 (Suppl 2):S285-S290.

Badi I, Burba I, Ruggeri C, Zeni F, Bertolotti M, and Scopece A, et al. (2015) MicroRNA-34a induces vascular smooth muscle cells senescence by SIRT1 downregulation and promotes the expression of age-associated pro-inflammatory secretory factors. J Gerontol A Biol Sci Med Sci 70:1304-1311.

Beltrami C, Angelini TG, and Emanueli C (2014) Noncoding RNAs in diabetes vascular complications. J Mol Cell Cardiol DOI: 10.1124/pr.55.3.4 [published ahead of print].

Blandino G, Valerio M, Cioce M, Mori F, Casadei L, Pulito C, Sacconi A, Biagioni F, Cortese G, and Galanti S, et al. (2012) Metformin elicits anticancer effects through the sequential modulation of DICER and c-MYC. Nat Commun 3:865.

Boon RA, Iekushi K, Lechner S, Seeger T, Fischer A, Heydt S, Kaluza D, Tréguer K, Carmona G, and Bonauer A, et al. (2013) MicroRNA-34a regulates cardiac ageing and function. Nature 495:107-110.

Caporali A, Meloni M, Völlenkle C, Bonci D, Sala-Newby GB, Addis R, Spinetti G, Losa S, Masson R, and Baker AH, et al. (2011) Deregulation of microRNA-503 contributes to diabetes mellitus-induced impairment of endothelial function and reparative angiogenesis after limb ischemia. Circulation 123:282-291.

Choi SE, Fu T, Seok S, Kim DH, Yu E, Lee KW, Kang Y, Li X, Kemper B, and Kemper JK (2013) Elevated microRNA-34a in obesity reduces NAD+ levels and SIRT1 activity by directly targeting NAMPT. Aging Cell 12:1062-1072.

Cui X, Chopp M, Zacharek A, Ye X, Roberts C, and Chen J (2011) Angiopoietin/Tie2 pathway mediates type 2 diabetes induced vascular damage after cerebral stroke. Neurobiol Dis 43:285-292.

Ding $\mathrm{H}$ and Triggle CR (2010) Endothelial dysfunction in diabetes: multiple targets for treatment. Pflugers Arch 459:977-994.

Ding J, Li M, Wan X, Jin X, Chen S, Yu C, and Li Y (2015) Effect of miR-34a in regulating steatosis by targeting $\operatorname{PPAR} \alpha$ expression in nonalcoholic fatty liver disease. Sci Rep 5:13729.

D’Onofrio N, Vitiello M, Casale R, Servillo L, Giovane A, and Balestrieri ML (2015) Sirtuins in vascular diseases: emerging roles and therapeutic potential. Biochim Biophys Acta 1852:1311-1322.

Eelen G, de Zeeuw P, Simons M, and Carmeliet P (2015) Endothelial cell metabolism in normal and diseased vasculature. Circ Res 116:1231-1244.

El-Mir MY, Nogueira V, Fontaine E, Avéret N, Rigoulet M, and Leverve X (2000) Dimethylbiguanide inhibits cell respiration via an indirect effect targeted on the respiratory chain complex I. J Biol Chem 275:223-228.

Fan W, Fang R, Wu X, Liu J, Feng M, Dai G, Chen G, and Wu G (2015) Shearsensitive microRNA-34a modulates flow-dependent regulation of endothelial inflammation. J Cell Sci 128:70-80.

Foretz M, Guigas B, Bertrand L, Pollak M, and Viollet B (2014) Metformin: from mechanisms of action to therapies. Cell Metab 20:953-966.

Fu T, Seok S, Choi S, Huang Z, Suino-Powell K, Xu HE, Kemper B, and Kemper JK (2014) MicroRNA 34a inhibits beige and brown fat formation in obesity in part by suppressing adipocyte fibroblast growth factor 21 signaling and SIRT1 function. Mol Cell Biol 34:4130-4142.

Fukuhara S, Sako K, Noda K, Zhang J, Minami M, and Mochizuki N (2010) Angiopoietin-1/Tie2 receptor signaling in vascular quiescence and angiogenesis. Histol Histopathol 25:387-396.

Giacco F and Brownlee M (2010) Oxidative stress and diabetic complications. Circ Res 107:1058-1070.

Halimi S (2006) Metformin: 50 years old, fit as a fiddle, and indispensable for its pivotal role in type 2 diabetes management. Diabetes Metab 32:555-556.

Han H, Qu G, Han C, Wang Y, Sun T, Li F, Wang J, and Luo S (2015) MiR-34a, miR21 and miR-23a as potential biomarkers for coronary artery disease: a pilot microarray study and confirmation in a 32 patient cohort. Exp Mol Med 47: e138

Hata A (2013) Functions of microRNAs in cardiovascular biology and disease. Annu Rev Physiol 75:69-93.

Ito T, Yagi S, and Yamakuchi M (2010) MicroRNA-34a regulation of endothelial senescence. Biochem Biophys Res Commun 398:735-740. 
Kinaan M, Ding H, and Triggle CR (2015) Metformin: an old drug for the treatment of diabetes but a new drug for the protection of the endothelium. Med Princ Pract 24: 401-415.

Kolluru GK, Bir SC, and Kevil CG (2012) Endothelial dysfunction and diabetes: effects on angiogenesis, vascular remodeling, and wound healing. Int $J$ Vasc Med 2012: 1-30.

Leeper NJ and Cooke JP (2011) MicroRNA and mechanisms of impaired angiogenesis in diabetes mellitus. Circulation 123:236-238.

Li X, Khanna A, Li N, and Wang E (2011) Circulatory miR34a as an RNA based, noninvasive biomarker for brain aging. Aging 3:985-1002.

Madiraju AK, Erion DM, Rahimi Y, Zhang XM, Braddock DT, Albright RA, Prigaro BJ, Wood JL, Bhanot S, and MacDonald MJ, et al. (2014) Metformin suppresses gluconeogenesis by inhibiting mitochondrial glycerophosphate dehydrogenase. Nature 510:542-546.

Mather KJ, Verma S, and Anderson TJ (2001) Improved endothelial function with metformin in type 2 diabetes mellitus. J Am Coll Cardiol 37:1344-1350.

Mattagajasingh I, Kim CS, Naqvi A, Yamamori T, Hoffman TA, Jung SB, DeRicco J, Kasuno K, and Irani K (2007) SIRT1 promotes endothelium-dependent vascular relaxation by activating endothelial nitric oxide synthase. Proc Natl Acad Sci USA 104:14855-14860.

Nisoli E, Tonello C, Cardile A, Cozzi V, Bracale R, Tedesco L, Falcone S, Valerio A Cantoni O, and Clementi E, et al. (2005) Calorie restriction promotes mitochondrial biogenesis by inducing the expression of eNOS. Science 310:314-317.

Olson EN (2014) MicroRNAs as therapeutic targets and biomarkers of cardiovascular disease. Sci Transl Med 6:239ps3.

Orimo M, Minamino T, Miyauchi H, Tateno K, Okada S, Moriya J, and Komuro I (2009) Protective role of SIRT1 in diabetic vascular dysfunction. Arterioscler Thromb Vasc Biol 29:889-894.

Owen MR, Doran E, and Halestrap AP (2000) Evidence that metformin exerts its anti-diabetic effects through inhibition of complex 1 of the mitochondrial respiratory chain. Biochem $J$ 348:607-614.

Pannirselvam M, Verma S, Anderson TJ, and Triggle CR (2002) Cellular basis of endothelial dysfunction in small mesenteric arteries from spontaneously diabetic ( $\mathrm{db} / \mathrm{db}-/-)$ mice: role of decreased tetrahydrobiopterin bioavailability. $\mathrm{Br} J$ Pharmacol 136:255-263.

Potente M and Dimmeler S (2008) Emerging roles of SIRT1 in vascular endothelial homeostasis. Cell Cycle 7:2117-2122.

Potente M, Ghaeni L, Baldessari D, Mostoslavsky R, Rossig L, Dequiedt F, Haendeler J, Mione M, Dejana E, and Alt FW, et al. (2007) SIRT1 controls endothelial angiogenic functions during vascular growth. Genes Dev 21:2644-2658.

Qin B, Yang H, and Xiao B (2012) Role of microRNAs in endothelial inflammation and senescence. Mol Biol Rep 39:4509-4518.

Roberts AC and Porter KE (2013) Cellular and molecular mechanisms of endothelial dysfunction in diabetes. Diab Vasc Dis Res 10:472-482.

Ruderman NB, Xu XJ, Nelson L, Cacicedo JM, Saha AK, Lan F, and Ido Y (2010) AMPK and SIRT1: a long-standing partnership? Am J Physiol Endocrinol Metab 298:E751-E760.

Semeniuk LM, Kryski AJ, and Severson DL (2002) Echocardiographic assessment of cardiac function in diabetic $\mathrm{db} / \mathrm{db}$ and transgenic db/db-hGLUT4 mice. Am $J$ Physiol Heart Circ Physiol 283:H976-H982.
Sena CM, Matafome P, Louro T, Nunes E, Fernandes R, and Seiça RM (2011) Metformin restores endothelial function in aorta of diabetic rats. $\mathrm{Br} \mathrm{J}$ Pharmacol 163:424-437.

Sena CM, Pereira AM, and Seiça R (2013) Endothelial dysfunction: a major mediator of diabetic vascular disease. Biochim Biophys Acta 1832:2216-2231.

Staszel T, Zapała B, Polus A, Sadakierska-Chudy A, Kieć-Wilk B, Stępień E, Wybrańska I, Chojnacka M, and Dembińska-Kieć A (2011) Role of microRNAs in endothelial cell pathophysiology. Pol Arch Med Wewn 121:361-366.

Suárez Y and Sessa WC (2009) MicroRNAs as novel regulators of angiogenesis. Circ Res 104:442-454.

Suri C, McClain J, Thurston G, McDonald DM, Zhou H, Oldmixon EH, Sato TN, and Yancopoulos GD (1998) Increased vascularization in mice overexpressing angiopoietin-1. Science 282:468-471.

Tabuchi T, Satoh M, Itoh T, and Nakamura M (2012) MicroRNA-34a regulates the longevity-associated protein SIRT1 in coronary artery disease: effect of statins on SIRT1 and microRNA-34a expression. Clin Sci 123:161-171.

Tremblay J and Hamet P (2015) Biomarkers of vascular complications in type 2 diabetes. Metabolism 64(Suppl 1):S28-S32.

Triggle CR and Ding H (2014) Cardiovascular impact of drugs used in the treatment of diabetes. Ther Adv Chronic Dis 5:245-268.

UK Prospective Diabetes Study Group (1998) Tight blood pressure control and risk of macrovascular and microvascular complications in type 2 diabetes: UKPDS 38. BMJ 317:703-713.

Urbich C, Kuehbacher A, and Dimmeler S (2008) Role of microRNAs in vascular diseases, inflammation, and angiogenesis. Cardiovasc Res 79:581-588.

Yamakuchi M, Ferlito M, and Lowenstein CJ (2008) miR-34a repression of SIRT1 regulates apoptosis. Proc Natl Acad Sci USA 105:13421-13426.

Yang Y, Cheng HW, Qiu Y, Dupee D, Noonan M, Lin YD, Fisch S, Unno K, Sereti KI, and Liao $\mathrm{R}$ (2015) MicroRNA-34a plays a key role in cardiac repair and regeneration following myocardial infarction. Circ Res 117:450-459.

Zampetaki A and Mayr M (2012) MicroRNAs in vascular and metabolic disease. Circ Res 110:508-522.

Zhang TX, Xu JX, Peng F, Chai DJ, and Lin JX (2013) Metformin reduces vascular endothelial dysfunction caused by an acute glucose load in patients with hypertension. Blood Press 22:106-113.

Zhao T, Li J, and Chen AF (2010) MicroRNA-34a induces endothelial progenitor cell senescence and impedes its angiogenesis via suppressing silent information regulator 1. Am J Physiol Endocrinol Metab 299:E110-E116.

Zheng Z, Chen H, Li J, Li T, Zheng B, Zheng Y, Jin H, He Y, Gu Q, and Xu X (2012) Sirtuin 1-mediated cellular metabolic memory of high glucose via the LKB1/ AMPK/ROS pathway and therapeutic effects of metformin. Diabetes 61:217-228.

Zhu $\mathrm{H}$ and Leung SW (2015) Identification of microRNA biomarkers in type 2 diabetes: a meta-analysis of controlled profiling studies. Diabetologia 58:900-911.

Address correspondence to: Dr. Hong Ding, Departments of Pharmacology and Medical Education, Weill Cornell Medicine-Qatar, P.O. Box \#24144, Education City, Doha, Qatar. E-mail: hod2005@qatar-med.cornell.edu 